\title{
Publisher Correction: Determining eigenstates and thermal states on a quantum computer using quantum imaginary time evolution
}

Mario Motta (1), Chong Sun, Adrian T. K. Tan, Matthew J. O’Rourke (1), Erika Ye, Austin J. Minnich (1),

Fernando G. S. L. Brandão and Garnet Kin-Lic Chan (D)

Correction to: Nature Physics https://doi.org/10.1038/s41567-019-0704-4, published online 11 November 2019.

In the version of this Article originally published online, in the first equation in the sentence beginning "One popular approach is", the term ' $-\partial_{\beta}|\Phi(\beta)\rangle=$ ' was repeated; the equation should have read " $-\partial_{\beta}|\Phi(\beta)\rangle=\left\{|\Phi\rangle, \hat{H}|\Phi\rangle, \hat{H}^{2}|\Phi\rangle \ldots\right\}|\Phi(\beta)\rangle$ ”. In the same sentence, in the expression " $\langle\Phi(0)| \Psi \neq 0$ ", the right ket bracket was missing and should have read ' $\langle\Phi(0) \mid \Psi\rangle \neq 0$ ". All versions of this Article have been amended.

Published online: 21 November 2019

https://doi.org/10.1038/s41567-019-0756-5

(c) The Author(s), under exclusive licence to Springer Nature Limited 2019

\section{Publisher Correction: Determining eigenstates and thermal states on a quantum computer using quantum imaginary time evolution}

Mario Motta (D), Chong Sun, Adrian T. K. Tan, Matthew J. O'Rourke (D), Erika Ye, Austin J. Minnich (D),

Fernando G. S. L. Brandão and Garnet Kin-Lic Chan (D)

Correction to: Nature Physics https://doi.org/10.1038/s41567-019-0704-4, published online 11 November 2019; corrected online 21 November 2019.

In the version of this Article originally published online, and in the later correction, the first equation in the sentence beginning "One popular approach is" was incorrect; the correct equation is ' $-\partial_{\beta}|\Phi(\beta)\rangle=\hat{H}|\Phi(\beta)\rangle$ '. This has now been amended in all versions of the Article.

Published online: 29 January 2020

https://doi.org/10.1038/s41567-020-0798-8

(c) The Author(s), under exclusive licence to Springer Nature Limited 2020

\section{Publisher Correction: Mathematical languages shape our understanding of time in physics}

Nicolas Gisin

Correction to: Nature Physics https://doi.org/10.1038/s41567-019-0748-5, published online 6 January 2020.

In the version of this Comment originally published online, the final sentence in Box 1 contained the erroneous text "numbers and stochastic"; it has now been removed.

Published online: 16 January 2020

https://doi.org/10.1038/s41567-020-0794-Z

(c) Springer Nature Limited 2020 